\title{
Fourier Methods for Oscillatory Differential Problems with a Constant High Frequency
}

\author{
L. Brugnano ${ }^{1}$, M. Calvo ${ }^{2}$, J.I. Montijano ${ }^{2, a)}$ and L. Rández ${ }^{2}$ \\ ${ }^{1}$ Dipartimento di Matematica e Informatica, Universita` di Firenze, Italy \\ ${ }^{2}$ IUMA-Departamento de Matem'atica Aplicada, Universidad de Zaragoza, Spain



\begin{abstract}
The numerical solution of highly oscillatory initial value problems of second order with a unique high frequency is considered. New methods based on Fourier approximations are proposed. These methods can integrate the problems with reasonable stepsizes not dependent on the size of the frequency.
\end{abstract}

\section{INTRODUCTION}

In this paper we consider the numerical integration of highly oscillatory initial value problems of the form

$$
\begin{aligned}
& y^{\prime \prime}(t)=\left(\begin{array}{cc}
-\omega^{2} I & 0 \\
0 & 0
\end{array}\right) y(t)+f(y(t)), \\
& y(0)=y_{0}, y^{\prime}(0)=y_{0}^{\prime} \in \mathbb{R}^{m},
\end{aligned}
$$

where $\omega \gg 1$ is large and $f$ is a smooth function of small size compared to $\omega^{2} y(t)$.

A lot of effort has been devoted to these class of equations $[3,14,9,2,8,6,5,10,11,1]$ considering different approaches. This type of problems present difficulties in their integration due to stability requirements and also accuracy requirements. To illustrate it, let us consider the scalar test problem

$$
y^{\prime \prime}=-\omega^{2} y+y^{3}, \quad y(0)=1 / 2, y^{\prime}(0)=1, \quad t \in[0,4]
$$

where we will take $\omega=100$ and $\omega=1000$. We will integrate it with the following three methods:

- The explicit RK of Bogacki and Shampine of order 3 [4],

- The Runge-Kutta Gauss-Legendre of order 2. It is A-stable and P-stable.

- The method based on Constant Variation Formula, due to Deuflhard [7], of order 2 which is symmetric and symplectic.

In Figure 1 we plot the maximum global error along the integration given by the three methods versus the step size used in the integration.

We can notice from the Figure several facts: The explicit RK is not stable for $h \omega>1$ whereas the other two seem to be stable (Deuflhard method can not be stable for some particular values of the stepsize), the methods exhibit numerically their order only for $h \omega<1$ whereas for large step sizes they show at most order zero, and the global errors, for a given step size, increase as $\omega$ increases. 




FIGURE 1. Errors given by the explicit RK method of order 3, RK Gauss formula of order 2 and Deufhlard method of order 2 for several values of the step size $h$

\section{A NEW APPROACH}

Consider first the initial value problem $y^{\prime \prime}=-\omega^{2} y+f(y), y(0)=y_{0}, y^{\prime}(0)=y_{0}^{\prime}, t \in[0, T]$, where we assume that $h \omega>1$. Using the constant variation formula, the solution can be expressed by means of the implicit equation

$$
y(t)=y_{0} \cos (\omega t)+\frac{y_{0}^{\prime}}{\omega} \sin (\omega t)+\int_{0}^{t} \frac{\sin (\omega(t-s))}{\omega} f(y(s)) \mathrm{d} s
$$

Using a functional iteration we get the sequence of approximations

$$
\begin{aligned}
& u^{[0]}(t)=y_{0} \cos (\omega t)+\frac{y_{0}^{\prime}}{\omega} \sin (\omega t), \\
& u^{[j+1]}(t)=u^{[0]}(t)+\int_{0}^{t} \frac{\sin (\omega(t-s))}{\omega} f\left(u^{[j]}(s)\right) \mathrm{d} s .
\end{aligned}
$$

They provide us a sequence of numerical approximations to the solution at the point $t=h$ by

$$
\begin{aligned}
& u^{[1]}(h)=u^{[0]}(h)+h \int_{0}^{1} \frac{\sin (\omega(1-\tau) h)}{\omega} f\left(u^{[0]}(\tau h)\right) \mathrm{d} \tau, \\
& u^{[j+1]}(h)=u^{[0]}(h)+h \int_{0}^{1} \frac{\sin (\omega(1-\tau) h)}{\omega} f\left(u^{[j]}(\tau h)\right) \mathrm{d} \tau .
\end{aligned}
$$

Assuming that $f(y)$ satisfies a Lipschitz condition $\|f(y)-f(z)\| \leq L\|y-z\|$, we have

Theorem 0.1 The approximations $u^{[j]}(h)$ satisfy

$$
\left\|y(h)-u^{[j]}(h)\right\| \leq \frac{h^{j+1}}{\omega^{j+1}} L^{j}\|f\|, \quad\left\|y^{\prime}(h)-\frac{d}{d t} u^{[j]}(h)\right\| \leq \frac{h^{j+1}}{\omega^{j}} L^{j}\|f\|
$$

\section{FOURIER METHODS}

The approximations in the above section give us numerical methods to compute the solution of the differential system if we are able to compute efficiently the quadratures

$$
\int_{0}^{t} \frac{\sin (\omega(t-s))}{\omega} f\left(u^{[j]}(s)\right) \mathrm{d} s
$$


Thus, to get $u^{[1]}$, we must compute the integral

$$
I\left(\omega, u^{[0]}\right)=\int_{0}^{t} \frac{\sin (\omega(t-s))}{\omega} f\left(y_{0} \cos (\omega s)+\frac{y_{0}^{\prime}}{\omega} \sin (\omega s)\right) \mathrm{d} s
$$

Since $\omega$ is supposed to be large, standard quadrature formulas will require a lot of nodes, unless $t \omega<2 \pi$ and this implies that the stepsize $h$ must be of the order of $1 / \omega$, which can be very small. Given the form of the integral, one can think about using Filon formulas $[12,13]$. However, this can not be useful enough because $f\left(u^{[0]}\right)$ is also highly oscillatory and can not be properly approximated by a Taylor polynomial unless $t$ is small.

Since $f\left(u^{[0]}\right)$ is periodic we can use a Fourier approximation

$$
f\left(u^{[0]}(t)\right) \simeq a_{0}+\sum_{n=1}^{k}\left[a_{n} \cos (n \omega t)+b_{n} \sin (n \omega t)\right]
$$

and the integral can be approximated by

$$
I\left(\omega, u^{[0]}\right) \simeq I_{k}\left(\omega, u^{[0]}\right)=\frac{1}{\omega} \sum_{n=0}^{k} a_{n} \int_{0}^{t} \sin ((t-s) \omega) \cos (n \omega s) \mathrm{d} s+\frac{1}{\omega} \sum_{n=1}^{k} b_{n} \int_{0}^{t} \sin ((t-s) \omega) \sin (n \omega s) \mathrm{d} s
$$

The integrals here can be expressed in terms of the trigonometric functions

$$
\begin{aligned}
& \int_{0}^{t} \frac{\sin (\omega(t-s))}{\omega} \cos (\omega s) \mathrm{d} s=\frac{1}{2 \omega} t \sin (\omega t), \quad \int_{0}^{t} \frac{\sin (\omega(t-s))}{\omega} \sin (\omega s) \mathrm{d} s=\frac{1}{2 \omega^{2}} \sin (\omega t)-\frac{1}{2 \omega} t \cos (\omega t) \\
& \int_{0}^{t} \frac{\sin (\omega(t-s))}{\omega} \cos (n \omega s) \mathrm{d} s=\frac{\cos (\omega t)}{\left(n^{2}-1\right) \omega^{2}}-\frac{\cos (n \omega t)}{\left(n^{2}-1\right) \omega^{2}}, \quad \int_{0}^{t} \frac{\sin (\omega(t-s))}{\omega} \sin (n \omega s) \mathrm{d} s=\frac{n \sin (\omega t)}{\left(n^{2}-1\right) \omega^{2}}-\frac{\sin (n \omega t)}{\left(n^{2}-1\right) \omega^{2}}
\end{aligned}
$$

and after some calculations the integral can be put in the form

$$
I_{k}\left(\omega, u^{[0]}\right)=\hat{a}_{0}+\sum_{n=1}^{k}\left[\hat{a}_{n} \cos (n \omega t)+\hat{b}_{n} \sin (n \omega t)\right]+t\left[\hat{c}_{1} \cos (\omega t)+\hat{d}_{1} \sin (\omega t)\right]
$$

where the coefficients $\hat{a}_{n}, \hat{b}_{n}, \hat{c}_{1}$ and $\hat{d}_{1}$ are linear combinations of the coefficients $a_{n}, b_{n}$ of the Fourier approximation. It can be proved the following

Theorem 0.2 The approximation $u_{k}^{[1]}(h)$ obtained by substituting $f\left(u^{[0]}\right)$ by a Fourier approximation with $k$ terms satisfies

$$
\left\|y(h)-u_{k}^{[1]}(h)\right\| \leq \frac{c(k, \alpha)}{\omega^{2}}+\frac{h^{2}}{\omega^{2}} L\|f\|
$$

where $c(k, \omega)$ is a coefficient that contains the effect of truncating the Fourier series and tends exponentially to zero when $k \rightarrow \infty$.

This methods can be extended so that they can be applied to the complete problem (1) by using some TaylorFourier expansion. For brevity this is not included in this document.

\section{NUMERICAL EXAMPLES}

To test the performance of the proposed approach, we have integrated the test problem (2), using three terms in the Fourier approximation, $k=3$, and we have compared the method with the method of Deuflhard. In Figure 2 we plot, for both methods and $\omega=100,1000$ the maximum global error in the solution $y\left(t_{n}\right)$ versus the number of evaluation of the function $f(y)$ employed in the integration. It can be seen in the plot that the Fourier method can integrate the problem with stepsize $h$ such that $h \omega>1$, the errors decrease as $\omega$ increases and it is clearly more efficient than the method of Deuflhard. 


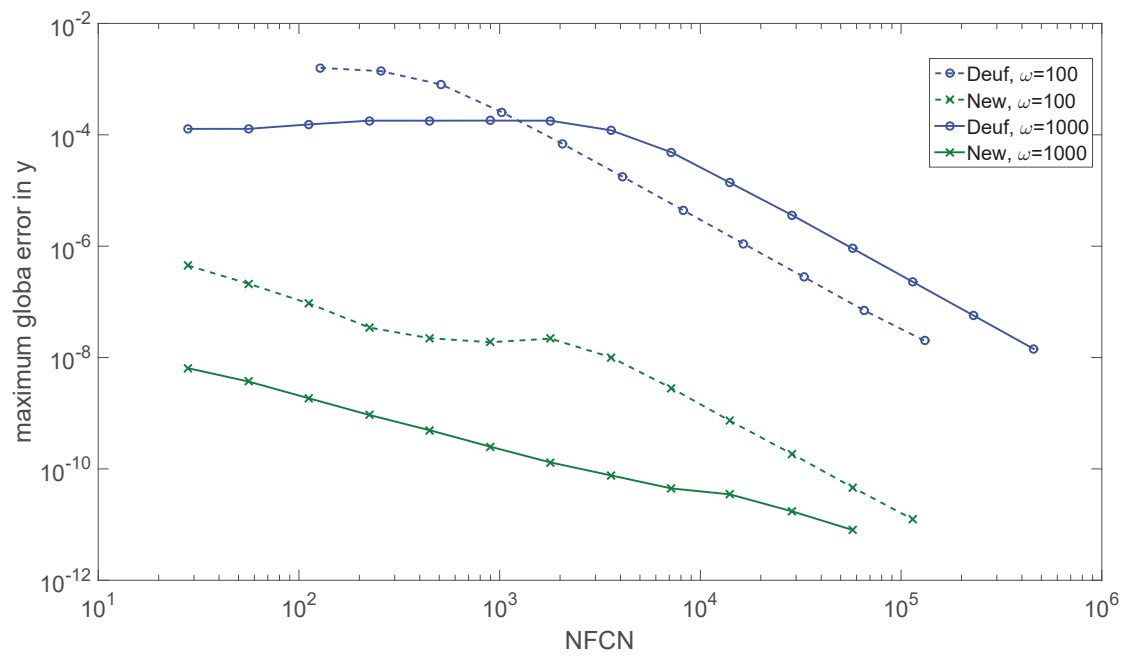

FIGURE 2. Errors given by the Fourier method and Deuflhard's method versus the number of evaluations of the non linear term $f$ for several values of the step size.

\section{ACKNOWLEDGMENTS}

This work was supported by project DGI MTM2013-47318-C2-1-P.

\section{REFERENCES}

[1] G. Ariel, B. Engquist, S. Kim, Y. Lee, and R. Tsai, A Multiscale Method for Highly Oscillatory Dynamical Systems Using a Poincaré Map Type Technique, J. Sci. Comput 54, 247-268 (2013).

[2] U. Ascher and S. Reich, "On some difficulties in integrating highly oscillatory hamiltonian systems," in Lect. Notes Comput. Sci., volume 4 (Springer, Berlin, 1999), pp. 281-296.

[3] D. G. Betis, Numerical Integration of Products of Fourier and Ordinary Polynomials, Numer. Math. 14, 421-434 (1970).

[4] P. Bogacki and L. F. Shampine, A 3(2) Pair of Runge-Kutta Formulas, Appl. Math. Leit. 2, 4, 321-325 (1989).

[5] M. Calvo, L. O. Jay, J. I. Montijano, and L. Rández, Approximate compositions of a near identity map by multi-revolution Runge-Kutta methods, Numer. Math. 4, 635-666 (2004).

[6] D. Cohen, "Analysis and numerical treatment of highly oscillatory differential equations," Ph.D. thesis, Université de Geneve 2004.

[7] P. Deuflhard, A Study of Extrapolation Methods Based on Multistep Schemes without Parasitic Solutions, Z. angew. Math. Phys. 48, 743-761 (1979).

[8] J. M. Franco, Runge-Kutta-Nyström methods adapted to the numerical integration of perturbed oscillators, Computer Physics Communications 30, 177-189 (2002).

[9] B. García-Archilla, J. M. Sanz-Serna, and R. D. Skeel, Long-time steps methods for oscillatory differential equations, SIAM J. Sci. Comput 20, 3, 930-963 (1998).

[10] E. Hairer, C. Lubich, and G. Wanner, Geometric Numerical Integration, 2nd ed. (Springer, Berlin, 2006).

[11] M. Hochbruck and A. Ostermann, Exponential integrators, Acta Numer. 19, 209-286 (2010).

[12] A. Iserles, S. P. Nørsett, and S. Olver, "Highly oscillatory quadrature: the story so far," in Numerical Mathematics and Advanced Applications (Springer, Berlin, 2006), pp. 97-118.

[13] M. Karnamiryanh, Quadrature Methods for Highly Oscillatory Linear and Nonlinear Systems of Ordinary Differential Equations: Part I, BIT Numerical Mathematics 48, 743-761 (2008).

[14] L. R. Petzold, L. O. Jay, and J. Yen, Numerical solution of highly oscillatory ordinary differential equations, Acta Numer. 6, 437-483 (1997). 